\title{
Evaluation of Anti-Oxidant Status and Radioprotective Activity of a Novel Anti-Cancer Drug in Mice
}

\author{
Raafat Yousri $^{1^{*}}$, Eman Noaman², Omama El Shawi², Nadia Fahmy², Maha Ghazy \\ ${ }^{1}$ Radiation Biology Department, National Centre for Radiation Research and Technology, Atomic Energy Authority, Cairo, Egypt; \\ ${ }^{2}$ Radiation Research and Health Department, National Centre for Radiation Research and Technology, Atomic Energy Authority, \\ Cairo, Egypt; ${ }^{3}$ Department of Zoology, Faculty of Science, El-Azhar University, Cairo, Egypt. \\ E-mail: raafatyousri@hotmail.com
}

Received September $20^{\text {th }}, 2011$; revised October $25^{\text {th }}, 2011$; accepted November $5^{\text {th }}, 2011$.

\begin{abstract}
Various approaches have been developed for diminishing the effects of radiation on normal tissues or enhancing tumor cell killing by ionizing radiation. Recently, there has been an increase in the interest in research on synthetic and/or natural radioprotective agents. An important potential use for these agents is to modify and improve the outcome of radiation therapy. The aim of this study was to examine the potential radioprotective role and antioxidant potency of the novel synthetic anticancer agent, quinoline sulfonamide (PIQSA) against tissue injury and oxidative stress induced by the exposure to gamma radiation and/or incidence of cancer in experimental animals. Mice (normal and bearing solid tumors) administered PIQSA $(0.350 \mathrm{mg} / \mathrm{kg}$ body weight ip. three times a week for 21 days. At the last week of 30 days experimental period, an animal group was subjected to three successive doses of $\gamma$-radiation each of 2 Gy; another group was treated with combined administration of PIQSA 20 minutes before $\gamma$-irradiation. Some biochemical parameters (LPx, GSH, SOD, and CAT in liver homogenates, also plasma lipid profile (total lipids, total cholesterol, total triglycerides (TG), HDLc and LDLc were measured. To examine any adverse effect which could be attained by chemical treatment, liver enzymes (AST, ALT), and kidney function (creatinine and urea) were estimated in blood plasma, in addition to examination of some haematological indexes. The results indicated that the deleterious effects due to exposure to $\gamma$-radiation, and/or incidence of cancer on most of the estimated parameters could be controlled to a certain extent by administration of PIQSA to animals prior to irradiation. The results also confirmed that there were no significant adverse effects on mice due to the treatment with this chemical compound.
\end{abstract}

Keywords: Quinoline Sulfonamide Derivative, Whole Body $\gamma$-IR, Radioprotector, Lipid Peroxidation, Antioxidants, Liver Enzymes, Kidney Function, Haematology

\section{Introduction}

Radiotherapy is an important modality for cancer treatment. It may be used as a single modality or an adjuvant along with the surgery and/or chemotherapy [1]. However, the effective use of ionizing radiation is comprised by side effects that result from the radiation induced damage to normal tissues [2]. Ionizing radiation causes harmful effects through the generation of free radicals.

Radiation damage is to a large extent caused by over production of reactive oxygen species (ROS) which cause disruption of membrane lipids leading to subsequent formation of peroxide radicals [3]. Moreover, Cancer cells have higher levels of reactive oxygen species
(ROS) than normal cells, and ROS are, in turn, responsible for the maintenance of the cancer phenotype. There is equilibrium between a free radical (FR)/reactive oxygen species (ROS) formation and endogenous antioxidant defence mechanisms, but if this balance is disturbed, it can produce oxidative stress. Oxidative stress, which is the imbalance between oxidant and antioxidants in favour of the oxidants, can result in injury to all the important cellular components like proteins, DNA and membrane lipids which can cause cell death [4]. Moreover, it was found that oxidative stress is associated with abnormal changes that may lead to disturbances in some physiological processes in the brain and liver that could render living organisms to be susceptible to infectious diseases 
and ultimately to death. Excessive generation of oxidants are overcoming body antioxidant capability in metabolizing them. These oxidants are often referred to as "free radical species". Free radicals include both Reactive Oxygen (ROS) and Nitrogen (RNS) Species and their production can be from endogenous as well as exogenous sources.

Against oxidative stress, cells are equipped with several natural enzymatic and nonenzymatic antioxidant defences [5]. A major defence mechanism involves the antioxidant enzymes including superoxide dismutase (SOD) and catalase (CAT) which convert active oxygen molecules into non-toxic compounds. The liver has the highest contents of antioxidants and antioxidant enzymes indicating that it plays an important role in pro-oxidants detoxification [6]. Wide spread use of radiation in diagnosis and therapy has necessitated development of a radioprotector to safeguard against human exposures. Pharmacological intervention could be the most prudent strategy [7] where a compound or formulation can act as free radical scavenger and antioxidant and reduce or mitigate the deleterious consequences of ionizing radiation. There is a continued interest in the identification and the development of nontoxic and effective radioprotectants that can reduce the effect of ionizing radiation [8]. The radioprotectors can elicit their action by various mechanisms such as 1) Suppressing the formation of free radicals. 2) Detoxifying the radiation induced reactive species. 3) Inducing the cellular radioprotectors such as superoxide dismutase (SOD), glutathione, prostaglandins and interleukine-1. 4) Enhancing the DNA repair by triggering one or more cellular DNA repair pathways and 5) delaying cell division by inducing hypoxia in tissues [9].

Recently, series of pyrimido quinoline compounds with sulfonamide moiety were synthesized and have been evaluated for their antioxidant and radioprotective efficacy [10]. Considering this point of view, the present work has been suggested to evaluate of the antioxidant potency and radioprotective efficacy of the novel synthetic compound,(4-[4-imino-8,8-dimethyl-6-oxo-3,5-diphenyl-2-thioxo-1,3,4,5,6,7,8,9-octahydro-2H-pyrimido[4, 5-b]quinolin-10yl]) bezenesulfonamide, (PIQSA).

\section{Materials and Methods}

\subsection{Chemicals}

A novel pyrimido-quinoline compound (PIQSA), bearing a sulfonamide moiety with molecular formula

$\left(\mathrm{C}_{31} \mathrm{H}_{29} \mathrm{~N}_{5} \mathrm{O}_{3} \mathrm{~S}_{2}\right)$.

Synthesis and analysis identification of the compound was previously published [10]. All other chemicals used in the present work were purchased from Sigma Chemical Company, USA.

\subsection{Animal Care and Handling}

The animal Care and Handling were done according to the guidelines issued by the World Health Organization. Geneva, Switzerland and approved from the committee for animal care at the National Centre for Radiation Research and Technology (NCRRT), Atomic Energy Authority (AEA). A total number of 100 old female Swiss albino mice weighing $30-35 \mathrm{~g}$ were selected for this study. Animals were maintained under controlled conditions of temperature $\left(23^{\circ} \mathrm{C}-25^{\circ} \mathrm{C}\right)$ with $12 \mathrm{~h}$ light and dark cycle. The animals were housed in cages with free access to drinking water and standard diet.

\subsection{Tumor Model}

A line of Ehrlish Ascite carcinoma cells (mouse tumors) that used during this work was supplied from the National Cancer Institute, Cairo, Egypt, was maintained and propagated by serial intraperitoneal transplantation of EAC cells in an aseptic environment. $10^{6}$ viable EAC cells were injected intraperitoneal into each animal in an aseptic condition and the day of tumor inoculation was considered as day zero. All the experiments were carried out on tumor bearing mice were conducted 10 days after the EAC transplantation and that day was considered as day one.

\subsection{Preparation of the Drug and Mode of Administration}

The chemical compound freshly prepared was dissolved in sterile DMSO/saline mixture and injected ip 10 days after transplantation of tumor cells into the mice.

Gamma irradiation facility was delivered through the NCRRT, Cairo, Egypt. Whole body gamma irradiation of animals was performed using Gamma cell-40 (Caesium137 source). Radiation exposure delivered three times each of 2 Gy at a dose rate of $0.85 \mathrm{G} / \mathrm{min}$ at the last week of the experimental period.

\subsection{Experimental Design and Treatments}

Three treatment modalities were applied during this work on both normal and also animals bearing tumors. Chemical treatment alone, treatment started at the $11^{\text {th }}$ day of the experimental period, animals of this group injected ip with $0.350 \mathrm{mg} / \mathrm{kg}$ body weight three times per week for 21 successive days. Radiation treatment alone, animals of this group subjected to the radiation doses at the last week of the experiment. Combined treatment modality, chemotherapy treatment started at the $11^{\text {th }}$ day of the experiment, animals injected with $0.350 \mathrm{~g}$ PIQSA $/ \mathrm{kg}$ body weight 20 minutes prior to the exposure to each of the gamma irradiation dose at the last week only. Animals were randomly divided into three main groups: Control 
group $(\mathrm{n}=20)$, normal treated animals $(\mathrm{n}=40)$, and animal bearing tumor group $(n=40)$. The normal treated group divided into four subgroups ( $\mathrm{n}=10$ of each), a: mice treated with DMSO, b: mice treated with PIQSA, c: mice subjected to gamma irradiation, d: mice received combined treatment of PIQSA followed by radiation treatment. The third main group, animal bearing tumors, this group also divided into four subgroups $(n=10$ in each) as the following; e: untreated animals bearing tumors ,f: animals bearing tumors treated with PIQSA, g: animals bearing tumors subjected to $\gamma$-irradiation, h: animal group received combined both $P I Q S A$ followed by $\gamma$-irradiation treatment. At the end of the experiment animals were kept fasting for 18 hours, thereafter, sacrificed, blood samples were collected into heparinized tubes, allowed standing for 15 minutes to clot at room temperature, then centrifuged using Heraeus Septech Centrifuge (Labofuge 200). The plasma was collected and kept at $-20^{\circ} \mathrm{C}$ till time of investigation. Liver was excised, rinsed in ice-cold normal saline followed by cold $0.15 \mathrm{~mol} / \mathrm{L}$ Tris- $\mathrm{HCl}$ buffer $(\mathrm{PH}=7.4)$, blotted dry, and weighed. A $10 \% \mathrm{w} / \mathrm{v}$ homogenate was prepared in $0.15 \mathrm{~mol} / \mathrm{L}$ Tris-HCl buffer and a portion was utilized for estimation of lipid peroxidation [11]. Another portion of liver homogenate was used for estimation of GSH content [12]. The supernatant was used for estimation of SOD [13] and catalase [14]. The plasma levels of total lipids [15], Total cholesterol [16], Total triglycerides [17] as well as plasma high density lipoprotein (HDL) [18] and plasma low density lipoprotein (LDL) [19] were measured. Also alanine aminotransferase (ALT) and aspartate aminotransferase (AST) activities [20] as well as creatinine [21] and urea levels [22] were estimated in plasma. About $1 / 2 \mathrm{ml}$ of whole blood was quickly taken into clean tubes mixed with anticoagulant and used freshly for blood counting, and haemoglobin estimation [23]. Statistical evaluation of the data was done using one way ANOVA test [24]. Data are shown as means \pm $\mathrm{SD}$ and the level for statistical significance was $\mathrm{p}<0.05$.

\section{Results}

Exposure of both normal group of animals and also animal group bearing tumors to three repeated doses of gamma radiation caused significant change in the different investigated parameters. While lipid peroxidation products in liver homogenate showed a significant elevation. On the contrary, inhibition of reduced glutathione (GSH) and both antioxidants SOD and CAT recorded in both irradiated animals groups (Tables 1 and 2). Administration of PIQSA to mice prior to IR exposure markedly ameliorated the elevation of lipid peroxidation and caused a marked improvement in GSH and SOD contents while insignificant change was observed in CAT content as compared to the control group (Tables 1 and 2). Our results also recorded significant elevation in all lipid fractions presented as total lipids, total cholesterol, total

Table 1. Lipid peroxide, glutathione content, SOD and CAT activity in liver of normal mice under different treatments.

\begin{tabular}{|c|c|c|c|c|}
\hline \multirow{2}{*}{ Treatments } & $\begin{array}{c}\text { Lipid peroxide } \\
\mu \mathrm{mol} \text { MAD/gm tissue }\end{array}$ & $\begin{array}{l}\text { GSH content } \\
\mathrm{Mg} / \mathrm{gm} \text { tissue }\end{array}$ & $\begin{array}{c}\text { SOD } \\
\mu \mathrm{g} / \mathrm{gm} \text { tissue }\end{array}$ & $\begin{array}{c}\text { CAT } \\
\mu \mathrm{mol} / \mathrm{gm} \text { tissue }\end{array}$ \\
\hline & \multicolumn{4}{|c|}{ Mean \pm SD and $\%$ of change from control group } \\
\hline Control & $82.31 \pm 3.18$ & $25.36 \pm 0.86$ & $30.7 \pm 5.024$ & $124.27 \pm 2.51$ \\
\hline \multirow{2}{*}{ DMSO } & $81.69 \pm 3.21^{\mathrm{NS}}$ & $25.14 \pm 0.68^{\mathrm{NS}}$ & $30.31 \pm 1.66^{\mathrm{NS}}$ & $124.45 \pm 2.97^{\mathrm{NS}}$ \\
\hline & $-0.75^{*}$ & $-0.87^{*}$ & $-1.27^{*}$ & $0.14^{*}$ \\
\hline \multirow{2}{*}{ PIQSA } & $83.19 \pm 1.91^{\mathrm{NS}}$ & $25.43 \pm 1.14^{\mathrm{NS}}$ & $32.82 \pm 3.56^{\mathrm{NS}}$ & $124.01 \pm 0.86^{\mathrm{NS}}$ \\
\hline & $1.07^{*}$ & $0.28^{*}$ & $6.91^{*}$ & $-0.21^{*}$ \\
\hline \multirow{2}{*}{ Irradiation } & $96.66 \pm 2.91^{\mathrm{a}}$ & $20.50 \pm 1.58^{\mathrm{a} 1}$ & $24.15 \pm 2.03^{\mathrm{a} 1}$ & $113.06 \pm 1.49^{\mathrm{a}}$ \\
\hline & $17.43^{*}$ & $-19.10^{*}$ & $-21.34^{*}$ & $-9.06^{*}$ \\
\hline \multirow{3}{*}{$\begin{array}{l}P I Q S A+ \\
\text { Irradiation }\end{array}$} & $88.93 \pm 1.21^{\mathrm{a}, \mathrm{b}}$ & $23.62 \pm 1.59^{\mathrm{a}, \mathrm{b}}$ & $25.64 \pm 2.58^{\mathrm{a}, \mathrm{b}}$ & $120.81 \pm 0.8^{\mathrm{NS}}$ \\
\hline & $8.04^{*}$ & $-6.69^{*}$ & $-6.05^{*}$ & $-2.78^{*}$ \\
\hline & $-9.200^{* *}$ & $15.02^{* *}$ & $18.24^{* *}$ & $6.85^{* *}$ \\
\hline
\end{tabular}

Mean of 5 animals/group. N.S = non significant; ${ }^{*}$ Percent change with respect to control group; ${ }^{* *}$ Percent change with respect $\gamma$-irradiation group; a: significance vs control group at $\mathrm{p}<0.05$; (a1): significance vs control group at $\mathrm{p}<0.01$; b: significance vs $\gamma$-irradiation group at $\mathrm{p}<0.05$. 
Table 2. Lipid peroxide, glutathione content, SOD and CAT activity in the liver of mice bearing tumors under different treatments.

\begin{tabular}{|c|c|c|c|c|}
\hline \multirow{2}{*}{ Treatments } & $\begin{array}{l}\text { Lipid peroxide } \\
\mu \text { mol/gm tissue }\end{array}$ & $\begin{array}{l}\text { GSH content } \mathrm{mg} / \mathrm{gm} \\
\text { tissue }\end{array}$ & $\begin{array}{c}\text { SOD } \\
\mu \mathrm{g} / \mathrm{gm} \text { tissue }\end{array}$ & $\begin{array}{c}\text { CAT } \\
\mu \mathrm{mol} / \mathrm{gm} \text { tissue }\end{array}$ \\
\hline & \multicolumn{4}{|c|}{ Mean \pm SD and $\%$ change from ESC group } \\
\hline Control & $82.31 \pm 3.18$ & $25.36 \pm 0.86$ & $30.70 \pm 5.024$ & $124.27 \pm 2.51$ \\
\hline \multirow{2}{*}{ ESC } & $91.19 \pm 1.28^{\mathrm{a}}$ & $23.07 \pm 1.27^{\mathrm{a}}$ & $25.72 \pm 2.47^{\mathrm{a} 1}$ & $119.18 \pm 0.80^{\mathrm{NS}}$ \\
\hline & $10.79^{*}$ & $-9.03 *$ & $-16.22^{*}$ & $-4.10^{*}$ \\
\hline \multirow{3}{*}{$\mathrm{ESC}+P I Q S A$} & $85.72 \pm 1.53^{\mathrm{NS}}$ & $23.37 \pm 1.01^{\mathrm{a}, \mathrm{bl}}$ & $27.83 \pm 2.12^{\mathrm{a}, \mathrm{b}}$ & $122.86 \pm 1.53^{\mathrm{b}}$ \\
\hline & $4.14^{*}$ & $-7.85^{*}$ & $-9.35^{*}$ & -0.92 \\
\hline & $-6.00^{* *}$ & $1.30^{* *}$ & $6.45^{* *}$ & $3.24^{* *}$ \\
\hline \multirow{3}{*}{ ESC + Irrad } & $99.21 \pm 2.19^{\mathrm{a} 1, \mathrm{~b}}$ & $16.37 \pm 1.034^{\mathrm{a} 2 \mathrm{~b}}$ & $18.22 \pm 2.08^{\mathrm{a} 2, \mathrm{~b}}$ & $116.75 \pm 0.72^{\mathrm{a}}$ \\
\hline & $20.53^{*}$ & $-35.45^{*}$ & $-40.65^{*}$ & $-6.05^{*}$ \\
\hline & $8.79^{* *}$ & $-29.04^{* *}$ & $-34.53^{* *}$ & $-5.58^{* *}$ \\
\hline \multirow{4}{*}{$\begin{array}{c}\mathrm{ESC}+\text { PIQSA } \\
\quad+\text { Irrad }\end{array}$} & $90.59 \pm 1.53^{\mathrm{a}, \mathrm{c}}$ & $21.61 \pm 1.98^{\mathrm{a} 1, \mathrm{~b}, \mathrm{c}}$ & $23.35 \pm 1.39^{\mathrm{al}, \mathrm{b}, \mathrm{cl}}$ & $121.05 \pm 1.17^{\mathrm{NS}}$ \\
\hline & $10.06^{*}$ & $-14.79^{*}$ & $-23.94^{*}$ & $-2.59^{*}$ \\
\hline & $-0.66^{* *}$ & $-7.37^{* *}$ & $-16.09^{* *}$ & $-1.69^{* *}$ \\
\hline & $-8.69^{* * *}$ & $-6.33^{* * *}$ & $28.16^{* * *}$ & $3.68^{* * *}$ \\
\hline \multirow{4}{*}{$\begin{array}{c}\mathrm{ESC}+\text { PIQSA } \\
+ \text { Irrad }\end{array}$} & $90.59 \pm 1.53^{\mathrm{a}, \mathrm{c}}$ & $21.61 \pm 1.98^{\mathrm{a} 1, \mathrm{~b}, \mathrm{c}}$ & $23.35 \pm 1.39^{\mathrm{a} 1, \mathrm{~b}, \mathrm{cl}}$ & $121.05 \pm 1.17^{\mathrm{NS}}$ \\
\hline & $10.06^{*}$ & $-14.79^{*}$ & $-23.94^{*}$ & $-2.59^{*}$ \\
\hline & $-0.66^{* *}$ & $-7.37^{* *}$ & $-16.09^{* *}$ & $-1.69^{* *}$ \\
\hline & $-8.69^{* * *}$ & $-6.33^{* * *}$ & $28.16^{* * *}$ & $3.68^{* * *}$ \\
\hline
\end{tabular}

Mean of 5 animals /group. N.S $=$ non significant. ${ }^{*}$ Percent changes with respect to control group. ${ }^{* *}$ Percent changes with respect to ESC group. ${ }^{* * *}$ Percent changes with respect to ESC $+\gamma$-irradiation group. a: significance vs control at $\mathrm{p}<0.05$. b: significance vs ESC group at $\mathrm{p}<0.05$, b1: significance vs ESC group at $\mathrm{p}<0.01$. c: significance vs ESC + irradiation group at $\mathrm{p}<0.05, \mathrm{c} 1$ :significance vs $\mathrm{ESC}+$ irradiation group at $\mathrm{p}<0.01$.

triglycerides and LDL-c, meanwhile a significant inhibition in HDL-c level in normal animal group subjected to $\gamma$-irradiation was recorded as compared to control group (Table 3). On the contrary a significant depression was recorded in the same parameters in the animals bearing tumor group except LDL-c level recorded a marked increase (Table 4). Administration of PIQSA to animals pre-IR markedly reduced the harmful effect of ionizing radiation on all measured lipid parameters investigated during this work as compared to either of control group or that exposed to $\gamma$-rays (Table 4). The activity of plasma AST \& ALT presented in (Tables 5 and 6) as well as of plasma creatinine and urea are illustrated in (Tables 6 and 7). A significant elevation in the activities of AST \& ALT observed post exposure of mice to $\gamma$ irradiation in both normal irradiated animals and animal bearing tumors when compared to the control group (Tables 5 and 6). The same result was also obvious in animal group bearing ESC tumors as compared to that of the control animals (Table 6). Moreover, treatment of mice with PIQSA before irradiation displayed a significant amelioration of the elevated enzymes activity as compared to that of the irradiated groups (Tables 5 and $\mathbf{6}$ ). Also, the same treatment reduced the level of creatinine and urea, which prove that $P I Q S A$ pre-treatment caused a marked protection against the increased level in creatinine and urea when compared to that of the irradiated group (Tables 6 and 7). In the present work, the effect of different treatments was also evaluated considering some blood indexes, the results were depicted in (Tables 9 and 10). The data revealed that there was a non significant change in the peripheral blood counts in normal animals treated with PIQSA (Table 9), indicating that this chemical compound has no adverse effect on the blood indexes at the level of the dose applied during these investigations. On the other hand, the significant improvement attained due to the same treatment in animal group bearing tumors (Table 10), may be an indication for its protective effect. Exposure of animal groups to $\gamma$-radiation induced significant decrease in $\mathrm{Hb}$ content as well as in 
RBCs and WBCs counts. Moreover, a significant change was recorded in the percentages of lymphocytes, monocytes and neutrophiles when compared to the control group (Tables 9 and 10). Administration of PIQSA pre-irradiation of mice minimized the harmful effect of ionizing radiation on the hemobiotic system as shown by the moderate increase in the measured blood parameters compared to those of the control animals or irradiated

Table 3. Effect of different treatments on plasma total lipids of normal mice.

\begin{tabular}{|c|c|c|c|c|c|}
\hline \multirow{2}{*}{ Treatments } & $\begin{array}{l}\text { Total Lipids } \\
\text { (mg/dl) }\end{array}$ & $\begin{array}{l}\text { T-Cholesterol } \\
\text { (mg/dl) }\end{array}$ & $\begin{array}{l}\text { Triglycerides } \\
\text { (mg/dl) }\end{array}$ & $\begin{array}{l}\text { HDL-Cholesterol } \\
(\mathrm{mg} / \mathrm{dl})\end{array}$ & $\begin{array}{l}\text { LDL-Cholesterol } \\
(\mathrm{mg} / \mathrm{dl})\end{array}$ \\
\hline & \multicolumn{5}{|c|}{ Mean $\pm \mathrm{SD}$ and $\%$ of changes } \\
\hline Control & $366 \pm 11.51$ & $61.47 \pm 6.38$ & $98.15 \pm 6.61$ & $34.38 \pm 3.53$ & $23.2 \pm 6.76$ \\
\hline \multirow{2}{*}{ DMSO } & $365.42 \pm 4.59^{\mathrm{NS}}$ & $62.32 \pm 6.37^{\mathrm{NS}}$ & $98.95 \pm 4.12^{\mathrm{NS}}$ & $34.13 \pm 1.1^{\mathrm{NS}}$ & $22.86 \pm 8.05^{\mathrm{NS}}$ \\
\hline & $-0.16^{*}$ & $1.38^{*}$ & $0.82^{*}$ & $-0.76^{*}$ & $-1.47^{*}$ \\
\hline \multirow{2}{*}{ PIQSA } & $348.3 \pm 6.14^{\mathrm{NS}}$ & $66.36 \pm 2.61^{\mathrm{NS}}$ & $91.98 \pm 7.63^{\mathrm{NS}}$ & $35.20 \pm 3.24^{\mathrm{NS}}$ & $21.04 \pm 4.42^{\mathrm{NS}}$ \\
\hline & $-4.83^{*}$ & $7.96^{*}$ & $-6.29^{*}$ & $2.39^{*}$ & $-9.31^{*}$ \\
\hline \multirow{2}{*}{ Irradiation } & $413.6 \pm 8.18^{\mathrm{a}}$ & $83.14 \pm 2.65^{\mathrm{a} 2}$ & $124.91 \pm 7.46^{\mathrm{a} 2}$ & $24.15 \pm 5.86^{\mathrm{a} 1}$ & $33.63 \pm 3.71^{\mathrm{a}}$ \\
\hline & $13.01^{*}$ & $35.25^{*}$ & $-27.26^{*}$ & $-29.76^{*}$ & $44.95^{*}$ \\
\hline \multirow{3}{*}{$P I Q S A+$ Irradiation } & $358.3 \pm 18.51^{b}$ & $58.34 \pm 3.62^{\mathrm{a}, \mathrm{b}}$ & $93.22 \pm 7.59^{\mathrm{b} 1}$ & $33.59 \pm 3.58^{\mathrm{b} 2}$ & $28.87 \pm 5.04^{\mathrm{a} 1, \mathrm{~b} 1}$ \\
\hline & $1.45^{*}$ & $9.54^{*}$ & $3.13^{*}$ & $3.52^{*}$ & $24.43^{*}$ \\
\hline & $-10.23^{* *}$ & $-19.004^{* *}$ & $-18.97^{* *}$ & $47.37^{* *}$ & $-14.15^{* *}$ \\
\hline
\end{tabular}

a: significance vs control at $\mathrm{p}<0.05$ and (a1) significance vs control at $\mathrm{p}<0.01$ and (a2) at $\mathrm{p}<0.001$. b: significance vs $\gamma$-irradiation group at $\mathrm{p}<0.05$, b1: significance vs $\gamma$-irradiation group at $\mathrm{p}<0.01, \mathrm{~b} 2$ : significance vs $\gamma$-irradiated group at $\mathrm{p}<0.001$.

Table 4. Effect of different treatments on plasma total lipids in ESC bearing mice.

\begin{tabular}{|c|c|c|c|c|c|}
\hline \multirow{2}{*}{ Treatments } & $\begin{array}{l}\text { Total Lipids } \\
(\mathrm{mg} / \mathrm{dl})\end{array}$ & $\begin{array}{l}\text { T-Cholesterol } \\
(\mathrm{mg} / \mathrm{dl})\end{array}$ & $\begin{array}{l}\text { Triglycerides } \\
\text { (mg/dl) }\end{array}$ & $\begin{array}{l}\text { HDL-Cholesterol } \\
(\mathrm{mg} / \mathrm{dl})\end{array}$ & $\begin{array}{l}\text { LDL-Cholesterol } \\
(\mathrm{mg} / \mathrm{dl})\end{array}$ \\
\hline & \multicolumn{5}{|c|}{ Mean \pm SD and $\%$ of changes } \\
\hline Control & $366 \pm 3.54$ & $61.28 \pm 6.38$ & $98.15 \pm 6.61$ & $31.38 \pm 3.53$ & $28.2 \pm 6.76$ \\
\hline \multirow{2}{*}{ ESC } & $323.6 \pm 13.90^{\mathrm{a}}$ & $49.59 \pm 9.52^{\mathrm{a}}$ & $83.91 \pm 5.42^{\mathrm{a}}$ & $24.35 \pm 4.49^{\mathrm{a}}$ & $34.02 \pm 2.91^{\mathrm{a}}$ \\
\hline & $-11.58^{*}$ & $-19.08^{*}$ & $-14.51^{*}$ & $-22.40^{*}$ & $20.64^{*}$ \\
\hline \multirow{3}{*}{$\mathrm{ESC}+P I Q S A$} & $333.6 \pm 13.9^{\mathrm{NS}}$ & $57.17 \pm 5.68^{\mathrm{a}, \mathrm{b}}$ & $88.92 \pm 7.64^{\mathrm{a}}$ & $28.41 \pm 4.63^{\mathrm{a}, \mathrm{b}}$ & $30.18 \pm 3.23^{\mathrm{b}}$ \\
\hline & $-8.85^{*}$ & $-6.70^{*}$ & $-9.40^{*}$ & $-9.45^{*}$ & $7.02^{*}$ \\
\hline & $3.09^{* *}$ & $15.28^{* *}$ & $5.97^{* *}$ & $16.76^{* *}$ & $-11.29^{* *}$ \\
\hline \multirow{3}{*}{$\mathrm{ESC}+$ Irradiation } & $264.25 \pm 16.03^{b}$ & $41.07 \pm 3.72^{\mathrm{b}}$ & $71.04 \pm 7.14^{\mathrm{a}, \mathrm{b}}$ & $44.95 \pm 2.56^{\mathrm{a}, \mathrm{b}}$ & $8.97 \pm 1.19^{\mathrm{a}, \mathrm{b}}$ \\
\hline & $-27.80^{*}$ & $-32.98^{*}$ & $-27.62^{*}$ & $43.24^{*}$ & $-68.19^{*}$ \\
\hline & $-18.34^{* *}$ & $-17.18^{* *}$ & $-15.34^{* *}$ & $84.60^{* *}$ & $-73.63^{* *}$ \\
\hline \multirow{4}{*}{$\begin{array}{l}\mathrm{ESC}+P I Q S A+ \\
\text { Irradiation }\end{array}$} & $348.64 \pm 7.38^{\mathrm{a}, \mathrm{b}, \mathrm{c}}$ & $46.56 \pm 5.14^{\mathrm{c}}$ & $78.32 \pm 6.12^{\mathrm{a}, \mathrm{c}}$ & $37.61 \pm 3.14^{\mathrm{a}, \mathrm{b}, \mathrm{c}}$ & $10.89 \pm 4.27^{\mathrm{a}, \mathrm{b}, \mathrm{c}}$ \\
\hline & $-4.73^{*}$ & $-24.02^{*}$ & -20.20 & $19.85^{*}$ & $-61.38^{*}$ \\
\hline & $7.74^{* *}$ & $-6.66^{* *}$ & $-6.66^{* *}$ & $54.46^{* *}$ & $-67.99^{* *}$ \\
\hline & $31.93^{* * *}$ & $13.36^{* * *}$ & $10.25^{* * *}$ & $-16.33^{* * *}$ & $21.40^{* * *}$ \\
\hline
\end{tabular}

a: significance vs control at $\mathrm{p}<0.05$ and (a1) significance vs control at $\mathrm{p}<0.001$. b: significance vs ESC bearing group at $\mathrm{p}<0.05$ and (b1) significance vs ESC group at $\mathrm{p}<0.01$. c: significance vs ESC bearing group + irradiation at $\mathrm{p}<0.05$. 
Table 5. Effect of different treatments on plasma AST \& ALT in normal mice.

\begin{tabular}{ccc}
\hline \multirow{2}{*}{ Treatments } & AST (U/L) & ALT (U/L) \\
\cline { 2 - 3 } & \multicolumn{2}{c}{ Mean \pm SD and \% of changes } \\
\hline ontrol & $39.2 \pm 1.25$ & $39.2 \pm 2.3$ \\
DMSO & $41 \pm 1.00^{\mathrm{NS}}$ & $41.40 \pm 1.1^{\mathrm{NS}}$ \\
& $4.59^{*}$ & $5.61^{*}$ \\
PIQSA & $42.40 \pm 2.10^{\mathrm{NS}}$ & $41.40 \pm 1.10^{\mathrm{NS}}$ \\
& $8.16^{*}$ & $5.61^{*}$ \\
Irradiation & $63 \pm 4.40^{\mathrm{a} 2}$ & $48.40 \pm 2.30^{\mathrm{a}}$ \\
& $60.71^{*}$ & $23.47^{*}$ \\
PIQSA + Irradiation & $55.40 \pm 4.51^{\mathrm{a} 2, \mathrm{~b}}$ & $44.20 \pm 3.70^{\mathrm{a}, \mathrm{b}}$ \\
& $41.33^{*}$ & $12.76^{*}$ \\
& $-12.06^{* * *}$ & $-8.68^{* * *}$ \\
\hline
\end{tabular}

a: significance vs control at $\mathrm{p}<0.05$ and a1 significance vs control at $\mathrm{p}<0.01$. b: significance vs $\gamma$-irradiation group at $\mathrm{p}<0.05$.

Table 6. Effect of different treatments on plasma AST \& ALT levels in mice bearing tumors.

\begin{tabular}{ccc}
\hline \multirow{2}{*}{ Treatments } & AST $(\mathrm{U} / \mathrm{L})$ & ALT $(\mathrm{U} / \mathrm{L})$ \\
\cline { 2 - 3 } & \multicolumn{2}{c}{ Mean \pm SD and $\%$ of changes } \\
\hline Control & $39.2 \pm 1.25$ & $39.2 \pm 2.3$ \\
ESC & $69 \pm 1.92^{\mathrm{a} 2}$ & $47.8 \pm 1.92^{\mathrm{a} 2}$ \\
& $76.02^{*}$ & $21.94^{*}$ \\
ESC+ PIQSA & $46.4 \pm 3.21^{\mathrm{a} 1}, \mathrm{~b} 2$ & $45.5 \pm 3.35^{\mathrm{a}}$ \\
& $18.06^{*}$ & $16.07^{*}$ \\
ESC+ Irrad & $-32.75^{* *}$ & $-4.81^{* *}$ \\
& $61 \pm 4.27^{\mathrm{a} 2 \mathrm{~b} 1}$ & $51.2 \pm 2.17^{\mathrm{a} 2, \mathrm{~b}}$ \\
& $55.61^{*}$ & $30.61^{*}$ \\
& $31.46^{* *}$ & $7.11^{* *}$ \\
ESC+ PIQSA+ Irrad & $53.8 \pm 1.30^{\mathrm{a} 2, \mathrm{~b} 2, \mathrm{c}}$ & $46.04 \pm 1.58^{\mathrm{a} 1, \mathrm{bl}, \mathrm{c}}$ \\
& $47.96^{* *}$ & $17.44^{*}$ \\
& $-32.03^{* *}$ & $17.45^{* *}$ \\
\hline
\end{tabular}

a: significance vs control at $\mathrm{p}<0.05$ and a2 significance vs control at $\mathrm{p}<0.001$. b: significance vs ESC bearing group at $\mathrm{p}<0.05$ and $\mathrm{b} 1$ significance vs ESC group at $\mathrm{p}<0.01$, c: significance vs ESC bearing group + irradiation at $\mathrm{p}<0.05$.

Table 7. Effect of the different treatments on plasma creatinine and urea levels of normal mice.

\begin{tabular}{ccc}
\hline \multirow{2}{*}{ Treatments } & Creatinine $(\mathrm{mg} / \mathrm{dl})$ & Urea $(\mathrm{mg} / \mathrm{dl})$ \\
\cline { 2 - 3 } & \multicolumn{2}{c}{ Mean \pm SD and \% of changes } \\
\hline Control & $0.91 \pm 0.1$ & $63.35 \pm 1.50$ \\
DMSO & $0.87 \pm 0.20^{\mathrm{NS}}$ & $67.90 \pm 3.02^{\mathrm{a}}$ \\
& $-4.39^{*}$ & $7.18^{*}$ \\
PIQSA & $0.86 \pm 3.20^{\mathrm{NS}}$ & $61.95 \pm 2.81^{\mathrm{NS}}$ \\
& $-5.49^{*}$ & $-2.21^{*}$ \\
Irradiation & $1.28 \pm 4.01^{\mathrm{a} 2}$ & $84.57 \pm 2.88^{\mathrm{a} 2}$ \\
& $40.66^{*}$ & $33.50^{*}$ \\
PIQSA + Irradiation & $1.14 \pm 3.22^{\mathrm{a} 1, \mathrm{~b}}$ & $69.59 \pm 1.50^{\mathrm{a}, \mathrm{b}}$ \\
& $25.27^{*}$ & $9.85^{*}$ \\
\hline
\end{tabular}

a: significance vs control at $\mathrm{p}<0.05$, a1 significance vs control at $\mathrm{p}<0.01$. b: significance vs $\gamma$-irradiation group at $\mathrm{p}<0.05$. 
Table 8. Effect of the different treatments on plasma creatinine and Urea levels in plasma of mice bearing tumors.

\begin{tabular}{ccc}
\hline \multirow{2}{*}{ Treatments } & Creatinine $(\mathrm{mg} / \mathrm{dl})$ & Urea $(\mathrm{mg} / \mathrm{dl})$ \\
\cline { 2 - 3 } Control & Mean \pm SD and $\%$ of changes \\
\hline ESC & $0.91 \pm 0.10$ & $63.35 \pm 1.5$ \\
& $1.09 \pm 0.10^{\mathrm{a} 1}$ & $75.30 \pm 1.4^{\mathrm{a} 1}$ \\
& $19.79^{*}$ & $18.68^{*}$ \\
ESC+PIQSA & $0.99 \pm 0.11^{\mathrm{a}, \mathrm{b}}$ & $68.93 \pm 1.70^{\mathrm{a}, \mathrm{b}}$ \\
& $8.79^{*}$ & $8.46^{*}$ \\
& $-9.17^{* *}$ & $-8.45^{* *}$ \\
ESC+ Irradiation & $1.33 \pm 0.10^{\mathrm{a} 2, \mathrm{bl}}$ & $83.27 \pm 1.10^{\mathrm{a} 2 \mathrm{~b}}$ \\
& $46.15^{*}$ & $31.44^{*}$ \\
& $22.08^{* *}$ & $10.58^{* *}$ \\
\hline
\end{tabular}

a: significance vs control at $\mathrm{p}<0.05$, a1 significance vs control at $\mathrm{p}<0.001$. b: significance vs ESC bearing group at $\mathrm{p}<0.05, \mathrm{~b} 1$ significance vs ESC group at $\mathrm{p}<0.01$. c: significance vs ESC bearing group + irradiation at $\mathrm{p}<0.01$.

Table 9. Changes in Haematological indexes in normal mice under different treatments.

\begin{tabular}{|c|c|c|c|c|c|c|}
\hline \multirow{2}{*}{ Treatments } & $\begin{array}{l}\mathrm{Hb} \\
(\mathrm{g} / \mathrm{dl})\end{array}$ & $\begin{array}{c}\text { RBCs } \\
\left(\times 10^{6} / \mathrm{ml}\right)\end{array}$ & $\begin{array}{c}\text { WBCs } \\
\left(\times 10^{3} / \mathrm{ml}\right)\end{array}$ & \% Lymphocytes & $\%$ Monocytes & $\%$ Neutrophiles \\
\hline & \multicolumn{6}{|c|}{ Mean $\pm \mathrm{SD}$ and $\%$ of changes } \\
\hline Control & $10.2 \pm 3.20$ & $7.38 \pm 1.76$ & $5.21 \pm 2.99$ & $81.00 \pm 6.20$ & $1.8 \pm 0.89$ & $23.6 \pm 5.13$ \\
\hline \multirow{2}{*}{ DMSO } & $10.12 \pm 1.18^{\mathrm{NS}}$ & $7.16 \pm 3.52^{\mathrm{NS}}$ & $5.16 \pm 3.12^{\mathrm{NS}}$ & $80.80 \pm 2.77^{\mathrm{NS}}$ & $1.80 \pm 1.14^{\mathrm{N} . \mathrm{S}}$ & $23.8 \pm 1.92^{\mathrm{NS}}$ \\
\hline & $-0.78^{*}$ & $-2.98^{*}$ & $-0.95^{*}$ & $-0.25^{*}$ & $0^{*}$ & $-0.85^{*}$ \\
\hline \multirow{2}{*}{$P I Q S A$} & $9.64 \pm 2.72^{\mathrm{NS}}$ & $7.05 \pm 2.60^{\mathrm{NS}}$ & $5.43 \pm 2.33^{\mathrm{NS}}$ & $83 \pm 4.95^{\mathrm{NS}}$ & $1.70 \pm 1.14^{\mathrm{N} . \mathrm{S}}$ & $24.2 \pm 1.92^{\mathrm{NS}}$ \\
\hline & $-5.49^{*}$ & $-4.47^{*}$ & $4.22^{*}$ & $2.47^{*}$ & $-5.55^{*}$ & $2.54^{*}$ \\
\hline \multirow{2}{*}{ Irradiation } & $9.02 \pm 3.23^{\mathrm{a}}$ & $6.60 \pm 4.17^{\mathrm{a}}$ & $4.73 \pm 1.91^{\mathrm{a}}$ & $73.44 \pm 7.73^{\mathrm{a}}$ & $1.40 \pm 1.09^{\mathrm{a} 1}$ & $16.6 \pm 1.10^{\mathrm{a} 1}$ \\
\hline & $-11.57^{*}$ & $-10.56^{*}$ & $-9.21^{*}$ & $-9.33^{*}$ & $-22.22^{*}$ & $-29.66^{*}$ \\
\hline \multirow{3}{*}{$P I Q S A+$ Irradiation } & $9.72 \pm 2.45^{\mathrm{b}}$ & $6.84 \pm 3.08^{\mathrm{NS}}$ & $5.09 \pm 2.14^{\mathrm{b}}$ & $78.80 \pm 9.83^{b}$ & $1.60 \pm 1.14^{\mathrm{a}, \mathrm{b} 1}$ & $21.20 \pm 2.58^{\mathrm{a}, \mathrm{b} 2}$ \\
\hline & $-4.71^{*}$ & $-7.32^{*}$ & $-2.30^{*}$ & $-2.72^{*}$ & $-11.11^{*}$ & $-10.17^{*}$ \\
\hline & $7.76^{* * *}$ & $3.64^{* * *}$ & $7.61^{* * *}$ & $14.53^{* * *}$ & $14.28^{* * *}$ & $27.21^{* * *}$ \\
\hline
\end{tabular}

$\mathrm{n}=5$ animals/experimental group. Combined treatment $=$ treatment with tested compound and $\gamma$-irradiation. N.S $=$ non significant. ${ }^{*}$ Represents percentage increase or decrease with respect to control value denoted by +ve and -ve signs respectively. ${ }^{* * *}$ Represents percentage increase or decrease with respect to normal animal group subjected to $\gamma$-irradiation. a: significance vs control. b: significance vs corresponding values in normal group treated with $\gamma$-irradiation. 
Table 10. Changes in Haematological indexes in mice bearing tumors under different treatments.

\begin{tabular}{|c|c|c|c|c|c|c|}
\hline \multirow{2}{*}{ Treatments } & $\begin{array}{l}\mathrm{Hb} \\
(\mathrm{g} / \mathrm{dl})\end{array}$ & $\begin{array}{c}\mathrm{RBCs} \\
\left(\times 10^{6} / \mathrm{ml}\right)\end{array}$ & $\begin{array}{l}\text { WBCs } \\
\left(\times 10^{3} / \mathrm{ml}\right)\end{array}$ & \% Lymphocytes & $\%$ Monocytes & $\%$ Neutrophils \\
\hline & \multicolumn{6}{|c|}{ Mean \pm SD and $\%$ of changes } \\
\hline Control & $10.2 \pm 3.20$ & $7.38 \pm 1.76$ & $5.21 \pm 2.99$ & $81.00 \pm 6.20$ & $1.8 \pm 0.89$ & $23.6 \pm 5.13$ \\
\hline \multirow{2}{*}{ ESC } & $8.45 \pm 2.07^{\mathrm{al}}$ & $6.05 \pm 2.51^{\mathrm{al}}$ & $6.2 \pm 3.22^{\mathrm{a} 1}$ & $65.2 \pm 11.1^{\mathrm{al}}$ & $1.6 \pm 2.03^{\mathrm{a}}$ & $30.2 \pm 1.9^{\mathrm{a} 1}$ \\
\hline & $-17.15^{*}$ & $-18.02^{*}$ & $19.01^{*}$ & $-19.51^{*}$ & $-11.11^{*}$ & $27.97^{*}$ \\
\hline \multirow{3}{*}{$\mathrm{ESC}+P I Q S A$} & $9.49 \pm 2.26^{\mathrm{b}}$ & $6.76 \pm 3.8^{b}$ & $5.8 \pm 2.67^{\mathrm{a}}$ & $73.20 \pm 13.4^{\mathrm{b}}$ & $1.7 \pm 2.49^{\mathrm{NS}}$ & $25.4 \pm 4.16^{\mathrm{a} 1}$ \\
\hline & $-6.96^{*}$ & $-8.40^{*}$ & $11.32^{*}$ & $-9.63^{*}$ & $-5.56^{*}$ & $7.63^{*}$ \\
\hline & $12.31^{* *}$ & $11.73^{* *}$ & $-6.45^{* *}$ & $12.27^{* *}$ & $-6.25^{* *}$ & $-15.89^{* *}$ \\
\hline \multirow{3}{*}{$\begin{array}{l}\mathrm{ESC}+\text { Irradia- } \\
\text { tion }\end{array}$} & $6.23 \pm 5.01^{\mathrm{a} 2, \mathrm{~b} 1}$ & $4.55 \pm 3.19^{\mathrm{a} 2, \mathrm{bl}}$ & $4.21 \pm 2.88^{\mathrm{a} 1, \mathrm{~b} 2}$ & $57.2 \pm 1.8^{\mathrm{a}, \mathrm{b}}$ & $1.00 \pm 1.73^{\mathrm{a} 2, \mathrm{~b} 2}$ & $24.6 \pm 4.28^{\mathrm{bl}}$ \\
\hline & $-38.92^{*}$ & $-38.3^{*}$ & $-19.19^{*}$ & $-29.38^{*}$ & $-44.44^{*}$ & $4.24^{*}$ \\
\hline & $-26.27^{* *}$ & $-24.79^{* *}$ & $-32.09^{* *}$ & $-12.27^{* *}$ & $-37.50^{* *}$ & $-18.54^{* *}$ \\
\hline \multirow{4}{*}{$\begin{array}{c}\mathrm{ESC}+\text { PIQSA+ } \\
\text { Irradiation }\end{array}$} & $8.77 \pm 2.28^{\mathrm{a}, \mathrm{c} 1}$ & $5.97 \pm 2.88^{\mathrm{a}, \mathrm{c} 1}$ & $4.54 \pm 0.20^{\mathrm{a}, \mathrm{bl}, \mathrm{c}}$ & $63.4 \pm 4.06^{\mathrm{a} 1, \mathrm{c}}$ & $1.4 \pm 1.14^{\mathrm{a} 1, \mathrm{~b}, \mathrm{c} 1}$ & $28.6 \pm 4.28^{\mathrm{a}, \mathrm{c}}$ \\
\hline & $-14.02^{*}$ & $-19.10^{* *}$ & $-12.85^{*}$ & $-21.73^{*}$ & $-22.22^{* *}$ & $21.19^{*}$ \\
\hline & $3.79^{* *}$ & $-1.32^{* *}$ & $-20.32^{* *}$ & $-2.76^{* *}$ & $12.50 * *$ & $-5.29^{* *}$ \\
\hline & $40.77^{* * *}$ & $31.21^{* * *}$ & $17.34^{* * *}$ & $10.84^{* * *}$ & $40^{* * *}$ & $16.26^{* * *}$ \\
\hline
\end{tabular}

NS = non significant. ${ }^{*}$ Represents percentage increase or decrease with respect to control value denoted by +ve and -ve signs respectively. ${ }^{* *}$ Represents percentage increase or decrease with respect to animal bearing tumors. ${ }^{* * *}$ Represents percentage increase or decrease with respect to normal animal group subjected to $\gamma$-irradiation. a: significance vs control at $\mathrm{p}<0.05$, a1 significance vs control at $\mathrm{p}<0.01$. b: significance vs ESC bearing group at $\mathrm{p}<0.05$, b1 significance vs ESC group at $\mathrm{p}<0.01, \mathrm{~b} 2$ significance vs ESC group at $\mathrm{p}<0.001$. c: significance vs ESC bearing group + irradiation at $\mathrm{p}<0.5$, c: significance vs ESC bearing group + irradiation at $\mathrm{p}<0.05$, c: significance vs ESC bearing group + irradiation at $\mathrm{p}<0.5$, c: significance vs ESC bearing group + irradiation at $\mathrm{P}<$ 0.01 .

\section{group (Table 9).}

\section{Discussion}

Ionizing radiation causes harmful effects through the generation of free radicals. When water, the most abundant intra and extracellular material, is exposed to ionizing radiation, decomposition occurs through which a variety of ROS, such as the superoxide radical, hydrogen peroxide $\left(\mathrm{H}_{2} \mathrm{O}_{2}\right)$ and the hydroxyl radical $\left(\mathrm{OH}^{-}\right)$are generated. These ROS formed in cells contribute to radiation injury in cells. Although all respiring cells are equipped with protective enzymes such as SOD and CAT or GPX, increased oxidative stress in cells that stem from ionizing radiation may overwhelm the protective systems, leading to cell injury. SOD converts super-oxide anion radical to $\mathrm{H}_{2} \mathrm{O}_{2}$, thus decreasing the amount of and the formation of peroxynitrite anion $\left(\mathrm{ONOO}^{-}\right)$, a highly destructive product of the interaction between $\mathrm{O}_{2}$ and nitric oxide [25].

The present study was carried out to demonstrate the protective the role of the novel pyrimido-quinoline compound (PIQSA) against the deleterious effects of $\gamma$-irradiation in treated animals.

Lipid peroxides, formed by the attack of radicals on polyunsaturated fatty acid residues of phospholipids, can further react with redox metals finally producing mutagenic and carcinogenic malondialdehyde, 4-hydroxynonenal and other exocyclic DNA adducts [26].

Accumulation of MAD is believed to be a cause of cell membrane damage.MAD, and has been used as an index of oxidative damage [25], and reflect the severity of cells attack by free radicals [27]. The results revealed a highly significant increase in MAD level in both groups subjected to $\gamma$-radiation (normal group and animal group bearing tumors) (Tables 1 and 2). Also a significant elevation in MAD level was recorded in the group of animals bearing tumors (Table 2). Similar observations in other laboratories were reported [28,29]. MDA, the end product of lipid peroxidation (LPO) was reported to be higher in carcinomatous tissue than in non diseased organs. Increased lipid peroxidation would cause degeneration of tissues. Lipid peroxide formed in the primary site would be transferred through the circulation and provoke damage by propagating the process of lipid peroxidation [30]. Administration of PIQSA to animal bearing tumors (Table 2) and also prior to irradiation treatment (Tables 1 and 2) induced a significant reduction in the lipid per- 
oxidation process. Since LPO is a good marker of damage that occurs due to whole body irradiation, therefore, the decrease of LPO is a sign of the radioprotective action of PIQSA administration to animals prior to irradiation. Cells evolve several antioxidant defences, including repair and detoxifying enzymes, and small scavenger molecules, such as glutathione. The intracellular ROS-scavenging system includes SOD, glutathione peroxidase (GPx), peroxiredoxins (PRDXs), glutaredoxins, thioredoxins (TRXs), and catalase [31]. Glutathione, a non enzymatic antioxidant, is the most abundant endogenous thiol-containing tri-peptide present in millimolar concentrations in eukaryotic cells. It play a pivotal role in maintenance of balance of cellular redox-states, metabolism, transport, catalysis as coenzymes, maintenance of thiol moieties, ect. It acts as radical scavenger, due to redox active sulfhydryl group directly reacting with oxidants and transforms itself into oxidizing glutathione [32]. Evidences suggest that patients suffering from oxidative stressinduced pathological diseases have decreased glutathione oxidant levels. The decreased glutathione levels generally been considered as an index of increased formation of ROS, and subsequent glutathione depletion caused oxidative stress-induced cellular damage [32]. A significant depletion in GSH content was observed during this work in animal group bearing tumors (Table 2) and in both animal groups subjected to $\gamma$-IR (Tables 1 and 2). These changes are almost similar to those reported in earlier [30,33]. The observed reduction in GSH content in animal group bearing tumors may be due to the inhibition of GSH synthesis or due to the lack of amino acids required for GSH formation [34]. Moreover, the depletion in GSH after exposure of mice to gamma radiation may be due to reaction of GSH with free radicals resulting in the formation of thiol radicals that associate to produce GSSG. Further, normal synthesis/repair of GSH will be impaired due to damage to DNA and membranes [34].

The free radical scavenging system, SOD and catalase are present in all oxygen metabolizing cells and their function are to provide a defence against the potentially damaging reactions of superoxide and hydrogen peroxide [35].

In the present investigation, a significant decrease in hepatic SOD and CAT activity occured in animal bearing tumor group (Table 2) and in the two groups subjected to gamma IR (Tables 1 and 2). Similar findings were observed previously [36]. The decrease in SOD activity in EAC bearing mice might be due to loss of Mn-SOD activity in EAC cells and the loss of mitochondria, leading to a decrease in total SOD activity in the liver [35]. On the other hand, the decrease in the activity of CAT could be due to a feed back inhibition or oxidative inactivation of enzyme protein caused by ROS generation [37]. The current study recorded a significant decrease in CAT activity in irradiated rats. However, CAT is one of three families of primary antioxidant enzymes in mammalian cells which are critical to peroxide removal. Therefore, the recorded reduction in the enzymatic activity of CAT may be due to the increased utilization of this antioxidant to counteract lipid peroxidation production [38].

Several possible biochemical mechanisms can explain the significant decrease in the activity of the antioxidant enzymes SOD, CAT due to the whole body gamma irradiation of mice observed in the present study. It is possible to conclude that radiation exposure-induced cell membrane damage with consequent alterations in the dynamic permeability of membranes due to peroxidation, which was followed by the release of intracellular enzymes to the blood stream [39]. Also this finding might be attributed to the antioxidant enzymes utilization by the enhanced production of ROS [40]. Moreover, the significant decrease in the activity of liver antioxidant enzymes of irradiated animals could be due to an increase in lipid peroxides which can cross-link with amino group of protein to form intra and intermolecular cross-links thereby inactivating several membrane bound enzymes [41].

The administration of PIQSA to tumor bearing mice and/or the two animal groups exposed to $\gamma$-IR caused partial improvement in the SOD as compared to both control and irradiated groups respectively. While a significant elevation in CAT activity was recorded with the irradiated group.

The present study demonstrated that administration of PIQSA for 21 days pre-3 repeated doses of $\gamma$-IR (each at 2Gy) and the extended doses of PIQS along with $\gamma$-IR has significantly reduced in the level of lipid peroxidation associated with enhancement in the activity of antioxidant enzymes SOD and CAT as well as in the content of reduced glutathione in the liver of the treated animals. This could indicate its potency as an inhibitor of ESC and/or $\gamma$-IR induced intracellular oxidative stress and also revealed the potential role of PIQSA as an antioxidant and free radical scavenging agent.

Lipids are major cell membrane components essential for various biological functions including cell growth and division of normal and malignant tissues [42].

Lipids might be associated with cancers as they have an integral role in the maintenance of cell integrity. Although, raised lipids are strongly associated with the pathogenesis of coronary heart disease, researchers have also reported an association between plasma/serum lipids and lipoproteins with different types of cancers [43]. Also $\gamma$-radiation changes the lipid profile and increases lipid peroxidation [44].

In the present work, data collected from animal bear- 
ing tumor group showed significant decrease in plasma total lipids, total cholesterol, total triglycerides (TG), and HDL-c. This result is in agreement with that published in several prospective and retrospective studies on the inverse association of lipid profile and different cancers [42]. Also animal studies have shown that nicotine, a known tobacco carcinogen affect activity of enzymes responsible for lipid metabolism [45]. It also reported that LDL uptake and cholesterol biosynthesis decreased in liver of tumor bearing animals [46]. This significant decrease in total lipid in some malignant diseases is due to the increase in lipid peroxidation that increased the utilization of total lipids including total cholesterol, and TG for new membrane biogenesis [47,48]. Table 1 shows an increase in the level of total lipids, cholesterol; triglycerides, 1DL-cholesterol associated with a significant decrease in the level of HDL-cholesterol in normal animal group exposed to $\gamma$-IR. These finding are in accordance with others [5]. Irradiation induces hyperlipidemia through cell membrane destruction, enhancement of lipid metabolism, cholesterol release and triglycerides synthesis [49]. Free radicals destruct cell membranes and enhance cholesterol release and increase lipid peroxidation [50].

The hyperlipidaemic state observed that also observed after irradiation of mice could be attributed to the mobilization of fats from the adipose tissue to the blood stream [51], in addition to mitochondrial dysfunction [52]. Regarding to results depicted in Table 1, which indicate that the administration of PIQSA to animals prior to irradiation reduced to a great extent the deleterious effect of $\gamma$-IR on the lipid fractions of the irradiated rats, indicating that the compound may has antilipidemic property.

On its own, administration of PIQSA didn't exhibit any adverse effect on either of liver or kidney functions (Tables 5 and 7). A significant elevation in AST and ALT enzymes activity (Tables $\mathbf{5}$ and $\mathbf{6}$ ) as well as in creatinine and urea (Tables $\mathbf{7}$ and 8) was observed in the two animal groups subjected to $\gamma$-radiation. It seems that there is an association between radiation induced oxidative stress and elevated different lipid fractions, as well as radiation induced oxidative stress and elevation of AST and ALT and increased levels of creatinine and urea. This association might point out that irradiation induced liver and kidney damages $[53,5]$. The increase in both aminotransferase activities in animal group bearing tumors (Table 6) was stated by other investigators [54], who reported the elevation of both enzymes activity in patients with wide varieties of cancers. Also AST and ALT activity showed an elevation in plasma fibrosarcoma induced rats [55]. Pre-administration of PIQSA to mice at a dose level of $0.350 \mathrm{mg} / \mathrm{kg}$ ameliorated the hazardous effect of irradiation on of AST and ALT activity (Tables 5 and 6) and also on the increased levels of creatinine and urea (Ta- bles 7 and 8 ) when compared to the irradiated animals.

In the present study, the effect of the different treatments on some haematological indexes was evaluated and the results were depicted in (Tables 9 and 10). The decrease in blood counts recorded in animal group bearing tumor being in consistent with others [56]. Anaemia and mylosuppression are of the common problems encountered in cancer therapy. Anaemia occurring in tumor-bearing mice is mainly due to reduction in RBCs, or $\mathrm{Hb}$ production. This event might occur either due to iron deficiency or haemolytic or mylopathic conditions [57].

Also, the decrease in haemoglobin content and RBCs counts was recorded with irradiated mice during this investigation could be attributed to the impairment of cell division, obliteration of blood-forming organs, alimentary tract injury, depletion of factors needed for erythroblast differentiation and reticulocyte release from the bone marrow and the loss of cells from the circulation by haemorrhage or leakage through capillary walls and/or the direct destruction of mature circulating cells [58]. The significant reduction recorded in WBCs in animal group exposed to $\gamma$-radiation could be explained on the bases that irradiation exposure-induced leucopoenia which was a direct consequence of the lymphopenia and neutropenia [59]. Pre-administration of PIQSA to mice restored the levels of the investigated blood indices with consequent increases in $\mathrm{Hb}$ content, RBCs \& WBCs. Moreover, a significant improvement in the percentages of leukocyte defferential counts was occured when compared to either of control group or irradiated group.

\section{Conclusions}

According to the fore mentioned results, we can conclude that PIQSA exhibited antioxidant efficacy, possibly may due to its ability to trigger the endogenous GSH level, and also the two main antioxidants enzymes, SOD and CAT activity. This action was accompanied with a suppression of lipid peroxidation. Also, PIQSA possess a radioprotective activity against radiation induced injury on different tissue organs (liver, Kidney and hemobiotic system).

\section{REFERENCES}

[1] W. P. Hogle, "Cytoprotective Agents Used in the Treatment of Patients with Cancer," Seminars in Oncology Nursing, Vol. 23, No. 3, 2007, pp. 213-224.

[2] E. J. Hall, "Radiotherapy for the Radiologist," 5th Edition, Williams and Wilkins, Philadelphia, 2000.

[3] N. Rajapakse, M. M. Kim, E. Mendis and S. K. Kim, 'Inhibition of Free Radical Mediated Oxidation of Cellular Biomolecules by Carboxylated Chitoologosaccharides," Bioorganic \& Medicinal Chemistry, Vol. 15, No. 2, 2007, pp. 997-1003. doi:10.1016/j.bmc.2006.10.030 
[4] V. R. Tandon, S. Sharma, A. Mahajan and G. H. Bardi, "Oxidative Stress: A Novel Strategy in Cancer Treatment," JK Science, Vol. 7, No. 1, 2005, p. 48.

[5] M. A. El-Missiry, T. A. Fayed, M. R. El-Sawy and A. A. El-Sayed, "Ameliorative Effect of Melatonin against Gamma-Irradiation-Induced Oxidative Stress and Tissue Injury," Ecotoxicology and Environmental Safety, Vol. 66 No. 2, 2007, pp. 278-286. doi:10.1016/i.ecoenv.2006.03.008

[6] J. A. Jalal, K. A. Albuseeda, T. A. Kumosani, K. O. Aboulnaja and M. F. Elshal, "Elucidation of the Determinant Factors Affecting Camels'Health in Some Regions of Saudi Arabia: A Biochemical, Histological, and Toxicological Study," Journal of King Abdulaziz University, Vol. 22, No. 1, 2010, pp. 225-238. doi:10.4197/Sci.22-1.15

[7] G. C. Jagetia, "Radioprotective Potential of Plants and Herbs against the Effects of Ionizing Radiation," Journal of Clinical Biochemistry and Nutrition, Vol. 40, No. 2, 2007, pp. 74-81. doi:10.3164/jcbn.40.74

[8] D. K. Chandrasekharan, T. V. Kagiya and C. K. K. Nair, "Radiation Protection by 6-Palmitoyl Ascorbic Acid-2Glucoside: Studies on DNA Damage in Vitro, ex Vivo, in Vivo and Oxidative Stress in Vivo," Journal of Radiation Research, Vol. 50, No. 3, 2009, pp. 203-212. doi:10.1269/jrr.08090

[9] D. K. Maurya, T. P. A. Devasagayam and C. K. Nair, "Some Novel Approaches for Radiation Protection and the Beneficial Effect of Natural Products," Indian Journal of Experimental Biology, Vol. 44, 2006, pp. 93-114.

[10] M. M. Ghorab, F. A. Ragab, E. Noaman, H. I. Heiba and E. M. El-Hossary, "Synthesis of Some Novel Quinolines and Pyrimido [4,5-b] Quinolines Bearing a Sulfonamide Moiety as Potential Anticancer and Radioprotective Agents," Arzneimittelforschung, Vol. 57, No. 12, 2007, pp. 795-803.

[11] T. Yoshioka, K. Kawada, T. Shimada and M. Mori, "Lipid Peroxidation in Maternal and Cord Blood and Protective Mechanism against Activated Oxygen Toxicity in the Blood," American Journal of Obstetrics \& Gynecology, Vol. 135, No. 3, 1979, pp. 372-376.

[12] E. Beutler, O. Duron and B. M. Kelly, "Improved Method for the Determination of Blood Glutathione," Journal of Laboratory and Clinical Medicine, Vol. 61, 1963, pp. 882-888.

[13] M. Minami and M. Yoshikawa, "A Simplified Assay Method of Superoxide Dismutase Activity for Clinical Use," Clinica Chimica Acta, Vol. 92, No. 3, 1979, pp. 337-342. doi:10.1016/0009-8981(79)90211-0

[14] L. H. Johansson and L. A. H. Borg, "A spectrophotometric Method for Determination of Catalase Activity in Small Tissue Samples," Analytical Biochemistry, Vol. 174, No. 1, 1988, pp. 331-336. doi:10.1016/0003-2697(88)90554-4

[15] J. A. Knight, S. Anderson and J. M. Rawle, "Estimation of Total Lipids in Serum and Tissues," Clinical Chemistry, Vol. 18, No. 3, 1972, pp. 199-202.
[16] D. Watson, "Simple Method for the Determination of Serum Cholesterol," Clinica Chimica Acta, Vol. 5, No. 5, 1960, pp. 637-643. doi:10.1016/0009-8981(60)90004-8

[17] P. Fossati and L. Principe, "Serum Triglycerides Determined Calorimetrically with an Enzyme That Produce Hydrogen Peroxide," Clinical Chemistry, Vol. 28 No. 10, 1982, pp. 2077-2080.

[18] G. R. Warnick, J. Benderson and J. J. Albers, "Dextran-Sulfate- $\mathrm{Mg}^{2+}$ Precipitation Procedure for Quantitation of High Density Lipoprotein Cholesterol," Clinical Chemistry, Vol. 28, No. 6, 1982, pp. 1397-1388.

[19] H. U. Bergmenyer, "Methods of Enzymatic Analysis," 3rd Edition, Vch Publisher, New York, 1985, pp. 154-160.

[20] S. Reitman and S. Frankel, "A Colorimetric Method for the Determination of Glutamic Oxalacetic and Glutamic Pyruvic Transaminases," American Journal of Clinical Pathology, Vol. 28, No. 1, 1957, pp. 56-63.

[21] R. J. Henry, D. C. Cannon and W. Winkelman, "Clinical Chemistry Principles and Techniques," 11th Edition, Happer and Row Publishers, New York, 1974.

[22] C. J. Patton and S. R. Crouch, "Spectrophotometric and Kinetics Investigation of the Berthelot Reaction for the Determination of Urea," Analytical Chemistry, Vol. 49, No. 3, 1977, pp. 464-469. doi:10.1021/ac50011a034

[23] J. V. Daci and S. M. Lewis, "Practical Haematology," 5th Edition, Churchill Living Stone, London, 1975.

[24] R. G. Steel and T. H. Torrie, "Principles and Procedures of Statistics," 2nd Edition, McCraw-Hill Book Co., New Delhi, 1980, p. 683.

[25] E Cicek, M. Yildiz, N. Delibas and S. Bahçeli, "The Effects of ${ }^{201} \mathrm{Tl}$ Myocardial Perfusion Scintigraphy Studies on Oxidative Damage in Patients," West Indian Medical Journal, Vol. 58, No. 1, 2009, pp. 50-53.

[26] H. Bartsch and J. Nair, "Potential Role of Lipid Peroxidation Derived DNA Damage in Human Colon Carcinogenesis: Studies on Exocyclic Base Adducts As Stable Oxidative Stress Markers," Cancer Detection and Prevention, Vol. 26, No. 4, 2002, pp. 308-312. doi:10.1016/S0361-090X(02)00093-4

[27] A. Valavanidis, T. Vlahoyianni and K. Fiotakis, “ Comparative Study of the Formation of Oxidative Damage Marker 8-hydroxy-2'-deoxyguanosine (8-OHdG) Adduct from the Nucleoside 2'-deoxyguanosine by Transition Metals and Suspensions of Particulate Matter in Relation to Metal Content and Redox Reactivity," Free Radical Research, Vol. 39, No. 10, 2005, pp. 1071-1081. doi:10.1080/10715760500188671

[28] M. Koc, S. Taysi, M. E. Buyukokuroglu and N. Bakan, "The Effect of Melatonin against Oxidative Damage during Total-Body Irradiation in Rats," Radiation Research, Vol. 160, No. 2, 2003, pp. 251-255. doi:10.1667/3034

[29] M. Sri Balasubashini, S. Karthigayan, S. T. Somasundaram, T. Balasubramanian, V. Viswanathan, P. Raveendran and V. P. Menon, "Fish Venom (Pterios volitans) Peptide Reduces Tumor Burden and Ameliorates Oxidative Stress in Ehrlich's Ascites Carcinoma Xenografted Mice," Bioorganic \& Medicinal Chemistry Letters, Vol. 
16, No. 24, 2006, pp. 6219-6225.

doi:10.1016/j.bmcl.2006.09.025

[30] M. Gupta, U. K. Mazumder, R. S. Kumar and T. S. Kumar, "Antitumor Activity and Antioxidant Role of Bauhinia Racemosa against Ehrlich Ascites Carcinoma in Swiss Albino Mice," Acta Pharmacologica Sinica, Vol. 25, No. 8, 2004, pp. 1070-1076.

[31] L. Gibellini, M. Pinti, M. Nasi, S. De Biasi, E. Roat, L. Bertoncelli and A. Cossarizza, "Interfering with ROS Metabolism in Cancer Cells: The Potential Role of Quercetin," Cancers, Vol. 2, No. 2, 2010, pp. 1288-1311. doi:10.3390/cancers2021288

[32] C. M. Panthak, P. K. Avti, S. Kumar, K. L. Khanduja and S. C. Sharma, "Whole Body Exposure to low Gamma Radiation Promotes Kidney Antioxidant Status in Balb/C Mice," Journal of Radiation Research, Vol. 48, No. 2, 2007, pp. 113-120. doi:10.1269/jrr.06063

[33] P. K. Avti, C. M. Pathak, S. Kumar, G. Kaushik, T. Kaushik, A. Farooque, K. L. Khanduja and S. C. Sharma. "Low Doses Gamma-Irradiation Differentially Modulates Antioxidant Defense in Liver and Lungs of Balb/C Mice," Journal of Radiation Research, Vol. 81, No. 12, 2005, pp. 901-910.

[34] J. Navarro, E. Obrador, J. Carretero, I. Petschen, J. Avino, P. Perez and J. M. Estrela, "Changes in Glutathione Status and the Antioxidant System in Blood and in Cancer Cells Associate with Tumour Growth in Vivo," Free Radical Biology \& Medicine, Vol. 26, 1999 pp. 410-418. doi:10.1016/S0891-5849(98)00213-5

[35] Y. Sun, L. W .Oberley, J. H. Elwell and E. Sierra-Rivera, "Antioxidant Enzyme Activities in Normal And Transformed Mice Liver Cells," International Journal of Cancer, Vol. 44, No. 6, 1989, pp. 1028-1033. doi:10.1002/ijc.2910440615

[36] Naveena, B. K. Bharath and S. Subramanian, "Antitumor Activity of Aloe Vera against Ehrlich Ascitis Carcinoma (EAC) in Swiss Albino Mice," International Journal of Pharma and Bio Sciences, Vol. 2, No. 2, 2011, pp. 400409

[37] Y .Ohta, M. Kongo-Nishimura, T. Matsura, K. Yamada, A. Kitagawa and T. Kishikawa, "Melatonin Prevents Disruption of Hepatic Reactive Oxygen Species Metabolism in Rats Treated with Carbon Tetrachloride," Journal of Pineal Research, Vol. 36, No. 1, 2004, pp. 10-17. doi:10.1046/j.1600-079X.2003.00091.X

[38] C. Kalpana and V. P. Menon, "Modulatory Effects of Curumin on Lipid Peroxidation and Antioxidant Status during Nicotine Induced Toxicity," Polish Journal of Pharmacology, Vol. 56, No. 5, 2004, p. 581.

[39] H. N. Saada, U. Z. Said and A. M. Mahdy, "Effectiveness of Aloe Vera on the Antioxidant Status of Different Tissues in Irradiated Rats," Pharmazie, Vol. 58, No. 2, 2003, pp. 929-931.

[40] U. Z. Said, K. S. Azab and S. M. Soliman, "Cardioprotective Role of Garlic (Allium sativum) on Oxidative Stress Induced by Gamma Radiaiton Exposure," Isotope and Radiation Research, Vol. 36, No. 3, 2004, pp. 465-479.
[41] T. Zima, L. Fialova, O. Mestek, M. Janebova, J. Crkovska, I. Malbohan, S. Stipek, L. Mikulikova and P. Popov, "Oxidative Stress, Metabolism of Ethanol and Alcohol-Related Diseases," Journal of Biomedical Science, Vol. 8, No. 1, 2001, pp. 59-70. doi:10.1007/BF02255972

[42] P. S. Patel, M. H. Shah, F. P. Jha, G. N. Raval, R. M. Rawal, M. M. Patel, J. B. Patel and D. D. Patel, "Alterations in Plasma Lipid Profile Patterns in Head and Neck Cancer and Oral Precancerous Conditions," Indian Journal of Cancer, Vol. 41, No. 1, 2004, pp. 25-31.

[43] A. Bielecka-Dąbrowa, S. Hannam, J. Rysz and M. Banach, "Malignancy-Associated Dyslipidemia," Open Cardiovascular Medicine Journal, Vol. 5, 2011, pp. 35-40. doi: $10.2174 / 1874192401105010035$

[44] S. K. Ardestani1, M. M. Janlow, A. Kariminia and Z. Tavakoli, "Effect of Cimetidine and Ranitidine on Lipid Profile and Lipid Peroxidation in $\gamma$-Irradiated Mice," Acta Medica Iranica, Vol. 42, No. 3, 2004, pp. 198-204.

[45] L. Ashakumary and P. L. Vijayammal, "Effect of Nicotine on Lipoprotein Metabolism in Rats," Lipids, Vol. 32, No. 3, 1997, pp. 311-315. doi:10.1007/s11745-997-0038-8

[46] P. Hermanik and L. H. Sobin, "International Union against Cancer (UICC): TNM Classification of Malignant Tumours, Head and Neck Tumours," 4th Edition, Springer, Geneva, 1987.

[47] M. A. Choi, B. S. Kim and R. Yu, "Serum Antioxidative vitamin Levels and Lipid Peroxidation in Gastric Carcinoma Patients," Cancer Letters, Vol. 136, No. 1, 1999, pp. 89-93. doi:10.1016/S0304-3835(98)00312-7

[48] O. E. Odeleye, C. D. Eskelson, S. I. Mufti and R. R. Watson, "Vitamin E Inhibition of Lipid Peroxidation and Ethanol-Mediated Promotion of Esophageal Tumorigenesis," Nutrition and Cancer, Vol. 17, No. 3, 1992, pp. 223 234. doi:10.1080/01635589209514191

[49] D. Applebaum-Bowden, P. McLean, A. Steinmetz, D. Fontana, C. Matthys, G. R. Warnick, M. Cheung, J. J. Albers and W. R. Hazzard, "Lipoprotein, Apolipoprotein and Lipolytic Enzyme Changes Following Estrogen Administration in Postmenoposal Women," Lipid Research, Vol. 30, No. 2, 1989, p. 1895.

[50] M. Karbowink and R. J. Reiter, "Antioxidative Effect of Melatonin Inprotection against Cellular Damage Caused by Ionizing Radiation," Proceedings of the Society for Experimental Biology and Medicine, Vol. 225, No. 1, 2000, pp. 9-22. doi:10.1046/j.1525-1373.2000.22502.x

[51] T. Chajek-Shaul, G. Friedman, O. Stein, E. Shiloni, J. Etienne and Y. Stein, "Mechanism of the Hypertriglyceridemia Induced by Tumor Necrosis Factor Administration to Rats," Biochimica et Biophysica Acta, Vol. 1001, No. 30, 1989, pp. 316-324.

[52] N. R. Madamanchi and M. S. Runge, "Mitochondrial Dysfunction in Atherosclerosis," Circulation Research, Vol. 100, No. 4, 2007, pp. 460-473. doi:10.1161/01.RES.0000258450.44413.96

[53] A. Onody, C. Csonka, Z. Giricz and P. Ferdinandy, "Hyperlipidemia Induced by a Cholesterol-Rich Diet Leads to 
Enhanced Peroxynitrite Formation in Rat Hearts," Cardiovascular Research, Vol. 58, No. 3, 2003, pp. 663-670. doi:10.1016/S0008-6363(03)00330-4

[54] V. Palani, R. K. Senthilkumaran and S .Govindasamy, "Biochemical Evaluation of Antitumor Effect of Muthu Marunthu (a Herbal Formulation) on Experimental Fibrosarcoma in Rats," Journal of Ethnopharmacology, Vol. 65 , No. 3, 1999, pp. 257-265. doi:10.1016/S0378-8741(98)00159-7

[55] P. Jayamathi, V. Vishnupriya and S. K. Mohan, "Biochemical Effects of Plumbagin on Fibrosarcoma Induced Rats," International Journal of Pharma Sciences and Research (IJPSR), Vol. 1, No. 8, 2010, pp. 320-325.

[56] B. Rajkapoor, M. Sankari, M. Sumithra, J. Anbu, N. Harikrishnan, M. Gobinath, V. Suba and R. Balaji, "Antitumor and Cytotoxic Effects of Phyllanthus polypyllus on Ehrlich Ascites Carcinoma and Human Cancer Cell Lines," Bioscience, Biotechnology, and Biochemistry, Vol. 71, No. 9, 2007, pp. 2177-2183. doi:10.1271/bbb.70149

[57] H. C. Hogland, "Hematological Complications of Cancer Chemotherapy," Seminar in Oncology, Vol. 9, No. 1, 1982, pp. 95-102.

[58] A. M. Abouelella, Y. E. Shahein, S. S. Tawfik and A. M. Zahran, "Phytotherapeutic Effects of Echinacea purpurea in Gamma-Irradiated Mice," Journal of Veterinary Science, Vol. 8, No. 4, 2007, pp. 341-351. doi:10.4142/jvs.2007.8.4.341

[59] S. Mishima, K. Saito, H. Maruyama, M. Inoue, T. Yamashita, T. Ishida and Y. Gu, "Antioxidant and ImmunoEnhancing Effects of Echinacea purpurea," Biological \& Pharmaceutical Bulletin, Vol. 27, No. 7, 2004, pp. 1004 1009. doi:10.1248/bpb.27.1004 